\begin{tabular}{|c|l|}
\hline Title & $\begin{array}{l}\text { Realization of an extremely low reflectance surface based on InP porous nanostructures for application to } \\
\text { photoelectrochemical solar cells }\end{array}$ \\
\hline Author(s) & Sato, Taketomo; Y oshizawa, Naoki; Hashizume, Tamotsu \\
\hline Citation & $\begin{array}{l}\text { Thin Solid Films, 518(15), 4399-4402 } \\
\text { https://doi.org/40.1016/.tsf.2010.02.029 }\end{array}$ \\
\hline Issue Date & 2010-05-31 \\
\hline Doc URL & http://hdl.handle.net/2115/43140 \\
\hline Type & article(author version) \\
\hline File Information & TSF518-15_4399-4402.pdf \\
\hline
\end{tabular}

Instructions for use 


\title{
Realization of an extremely low reflectance surface based on InP porous nanostructures for application to photoelectrochemical solar cells
}

\author{
Taketomo Sato*, Naoki Yoshizawa, and Tamotsu Hashizume \\ Research Center for Integrated Quantum Electronics (RCIQE), Hokkaido University, \\ Sapporo, 060-8628, Japan
}

\begin{abstract}
Extremely low reflectance was obtained from InP porous nanostructures in UV, visible, and near-infrared ranges. Porous samples were electrochemically prepared on which 130-nm-diameter nanopores were formed in a straight, vertical direction and were laterally separated by $50-\mathrm{nm}$-thick InP nanowalls. The reflectance strongly depended on the surface morphology. The lowest reflectance of $0.1 \%$ in the visible light range was obtained after the irregular top layer had been completely removed. Superior photoelectrochemical properties were obtained on the InP porous structures due to two unique features: the large surface area inside pores, and the large photon absorption enhanced on the low reflectance surface.
\end{abstract}

Keywords: Indium Phosphide, porous structure, surface reflectance, photoelectrochemical solar cell

*Corresponding author. E-mail: taketomo@rciqe.hokudai.ac.jp (Taketomo Sato).

\section{Introduction}

Indium phosphide and related materials have attracted attention over the years as effective materials for high-speed electronics and optoelectronic devices. Photosensitive devices such as photodetectors in long-wavelength optical fiber communication systems $[1,2]$ and high-efficiency solar cells with multi-junction structures [3] are excellent examples of technologies that utilize the superb optical properties of InP-based systems. With the aforementioned devices, however, surface reflection is a serious problem that degrades the device performance because is reduces the efficiency of photon energy conversion [4]. Surface texturing of V-grooves [5-7], formation of insulator films [8], wide bandgap semiconductor films [9], and transparent conducting oxide films [10] have been investigated as anti-reflective layers on top of InP. However, most of these techniques exhibit minimum reflectance values as low as $2-10 \%$ and satisfactory anti-reflective performance only in a limited wavelength range.

In this letter, we report that extremely low reflectance below $0.4 \%$ was observed from the InP porous nanostructures in UV, visible, and near-infrared ranges. Our porous structures are electrochemically formed using a very simple setup at extremely low cost. The surface reflectance spectroscopy was systematically carried out on various porous samples, leading to finding that the reflectance strongly depended on the surface morphology and the pore depth. Photoelectrochemical measurements on the porous structures revealed that the photocurrents increased in the samples that had low reflectance surfaces with deeper pores.

\section{Experimental details}

The porous samples were electrochemically prepared as follows. The initial substrate consisted of a layer of (001) n-type InP $\left(\mathrm{n}=2-3 \times 10^{18} \mathrm{~cm}^{-3}\right)$, on the back of which a $\mathrm{GeAu} / \mathrm{Ni}$-ohmic contact was formed to supply current. The InP substrate was first anodized at $5 \mathrm{~V}$ in an HCl-based electrolyte to form the porous structures, where the structure depth, $d$, could be controlled by the anodization time, $t_{\mathrm{a}}[11]$. As schematically shown in Fig. 1(a), a disordered irregular layer formed and remained on top of the ordered porous layer after the first anodization. To remove this irregular layer, the porous surface was photo-electrochemically etched under illumination at an anodic bias of $1 \mathrm{~V}$ in the same

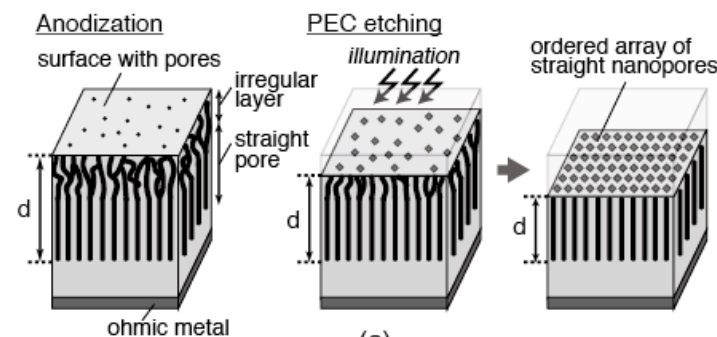

(a)

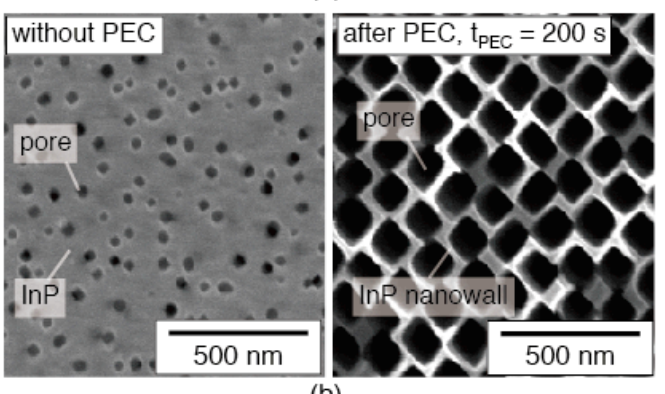

(b)

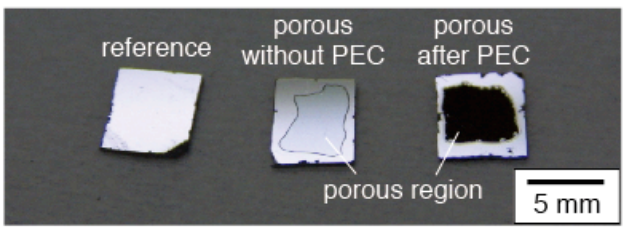

(c)

Figure 1: (a) Schematic illustration of anodized porous structures and procedure for subsequent PEC etching. (b) SEM images of porous samples formed without PEC etching $\left(t_{\mathrm{PEC}}=0 \mathrm{~s}\right)$ and after PEC etching $\left(t_{\mathrm{PEC}}=200 \mathrm{~s}\right)$. (c) Photograph of planar InP reference, porous sample without PEC etching, and porous sample after PEC etching. 
electrolyte [12]. Figure 1(b) shows typical scanning electron microscope (SEM) images of the top view of the InP porous samples just after anodization and after photo-electrochemical (PEC) etching for $200 \mathrm{~s}\left(t_{\mathrm{PEC}}=\right.$ $200 \mathrm{~s})$. The average pore diameter in the sample before the PEC etching was about $30 \mathrm{~nm}$. On the other hand, regular sized pores about $130 \mathrm{~nm}$ in diameter appeared after PEC etching with $t_{\mathrm{PEC}}=200 \mathrm{~s}$, which was useful in completely removing the disordered layer from the surface. From the SEM observation on the cross-section, it was found that 130 -nm-diameter nanopores were laterally separated by 50 -nm-thick InP nanowalls.

We found that the optical view of the porous surface was reflected in the differences in the microscopic surface morphology. As shown in Fig. 1(c), the PEC etched porous sample has diffuse optical reflection resulting in a black surface. In this study, the reflectance spectra of various samples with different pore depths, $d$, were compared in detail using an ultra-violet (UV)-visible spectrometer (UV-1700, Shimadzu). The pore depth, $d$, was controlled by changing the first anodization time, $t_{\mathrm{a}}$, and the PEC processing time, $t_{\mathrm{PEC}}$. The photon energy range of the light source was set from 1.12 to $6.0 \mathrm{eV}$, corresponding to the wavelength range from 205 to $1100 \mathrm{~nm}$. After that, photoelectrochemical measurements were conducted on the various porous InP electrodes under white light from a tungsten lamp. For the photocurrent measurements, $1 \mathrm{mM} \quad \mathrm{K}_{3} \mathrm{Fe}(\mathrm{CN})_{6}$ electrolyte was used. In this electrolyte system, n-type InP surface is relatively stable and a large potential barrier with the $\left[\mathrm{Fe}(\mathrm{CN})_{6}\right]^{3-/ 4-}$ redox potential is expectable [13]. For comparison, a planar InP electrode without a porous structure was prepared. Just before the electrochemical measurement, the surface of the InP planar electrode was also photo-electrochemically etched about $200 \mathrm{~nm}$ in order to remove the native oxides and damaged layers. This treatment was important and effectively improved the electrochemical activity of the InP electrode. The circular open windows were formed on all samples using a photolithography technique to define the geometrical area of the electrodes. The diameter and area of the circular windows were $6 \mathrm{~mm}$ and $0.28 \mathrm{~cm}^{2}$, respectively.

\section{Results and discussions \\ 3.1. Surface reflectance}

We first investigated the effects of the irregular top layer on the surface reflectance by comparing various porous samples formed with different $t_{\mathrm{PEC}}$ values, as shown in Fig. 2(a). The pore depth, $d$, of the sample just after anodization without PEC etching was $13.8 \mu \mathrm{m}$, including the $4.0-\mu \mathrm{m}$-thick irregular top layer. After the sample was PEC etched for $200 \mathrm{~s}$, the $d$ decreased to 9.6 $\mu \mathrm{m}$ due to the irregular layer having been removed. First, the reflectance of the planar sample was higher than $30 \%$ over the measurement range. Typical peaks were observed around $1.4 \mathrm{eV}, 3.0 \mathrm{eV}$, and 4.5-5.6 eV, which were attributed to the interband transitions [14]. The highest peak observed below $1.4 \mathrm{eV}$ was the reflection on the back ohmic contact, which acted as a mirror for light transmitted through the InP bandgap. The reflectance obtained from the porous sample without
PEC etching was 5-10\% lower than that obtained from the reference sample. As plotted in Fig. 2(a), the spectral features of the InP bulk remained in the porous sample, showing that crystal quality was maintained beyond a certain level after the pore had formed. On the other hand, the reflectance of the porous sample with the PEC etching drastically decreased compared with those of the other two samples. These results are very consistent with the optical view shown in Fig. 1(c).

Figure 2(b) compares the reflectance spectra obtained from the porous samples after being PEC etched as a function of the processing time, $t_{\mathrm{PEC}}$. From the SEM observation, the pore depth, $d$, of the sample was found to have decreased almost linearly with $t_{\mathrm{pec}}$, indicating that the irregular layer had been gradually etched from the top surface. As $t_{\mathrm{pec}}$ increased, the reflectance of the porous samples decreased, as shown in
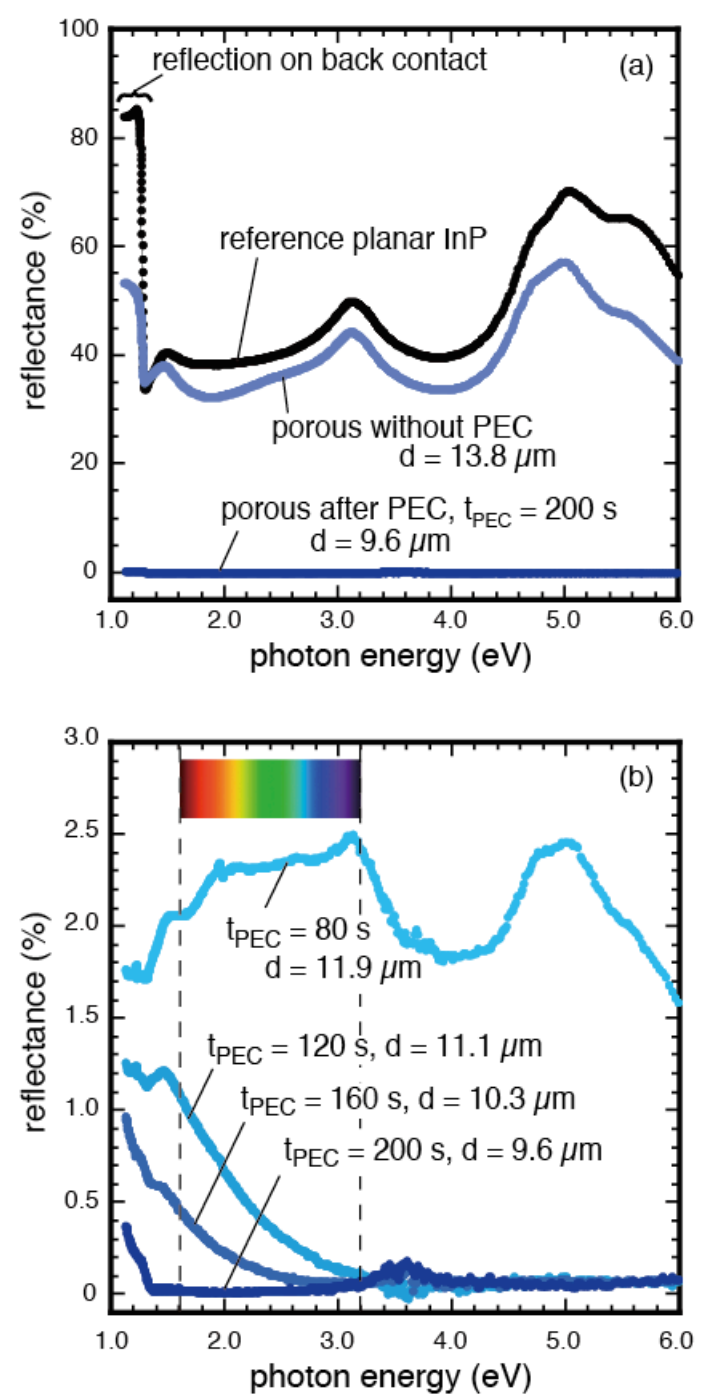

Figure 2: (a) Surface reflectance spectra measured as a function of photon energy of incident light for three samples shown in Fig. 1(c). (b) Surface reflectance spectra measured for porous samples after PEC etching with different $t_{\mathrm{PEC}}$. Irregular top layers still remained on three samples prepared with $t_{\mathrm{PEC}}=80,120$, and $160 \mathrm{~s}$ and were completely removed for sample prepared with $t_{\mathrm{PEC}}=$ $200 \mathrm{~s}$. 
T. Sato et al., Thin Solid Films, 518(15), 2010, pp. 4399-4402.

Fig. 2(b). For the sample with $t_{\mathrm{PEC}}=200 \mathrm{~s}$, where the irregular top layer was completely removed, the reflectance was lower than $0.4 \%$ over the measurement range. In the visible light range of 1.6-3.2 eV, which corresponds to the wavelength range of $380-750 \mathrm{~nm}$, the reflectance dropped to below $0.1 \%$, much lower than the values of 5-10\% reported for Si porous structures [15].

A possible explanation for the low reflectance observed here is that the straight nanopores with enlarged openings appeared on the surface after the PEC etching. In the present case with our porous samples, air holes are closely aligned between the InP nanowalls with a filling factor, $f$, of about 0.3 . This kind of air-dielectric composite, which when assembled in an ordered array, has a small $n$ close to unity leading to the low reflectance on the air interface. In other instances for similar nanostructures, an extremely low reflectance of $0.045 \%$ was observed from the vertically aligned carbon nanotubes where the small $n$ of 1.026 was expected [16]. On the other hand, it is possible that the irregular top layer has a large $n$ value due to the winding pores with poor porosity, resulting in the surface reflectance reducing by only a few percent.

\subsection{Photoelectrochemical properties}

To investigate the feasibility of applying the porous samples to high-efficiency solar cells, the photoelectrochemical measurements were conducted on them after the irregular top layer had been removed. Figure 3 shows the cyclic voltammograms obtained at the porous InP electrodes with $d=9.6 \mu \mathrm{m}$ in the $\mathrm{K}_{3} \mathrm{Fe}(\mathrm{CN})_{6}$ electrolyte under various light conditions. The current density was calculated using the geometrical surface area of $0.28 \mathrm{~cm}^{2}$ for all electrodes. As shown in Fig. 3, the cathodic currents changed little as the light power changed. However, the anodic currents increased systematically as the light power increased where the dark current showed good blocking behavior. These results indicate that the basic photovoltaic property of n-type semiconductors remained on the porous InP electrode.

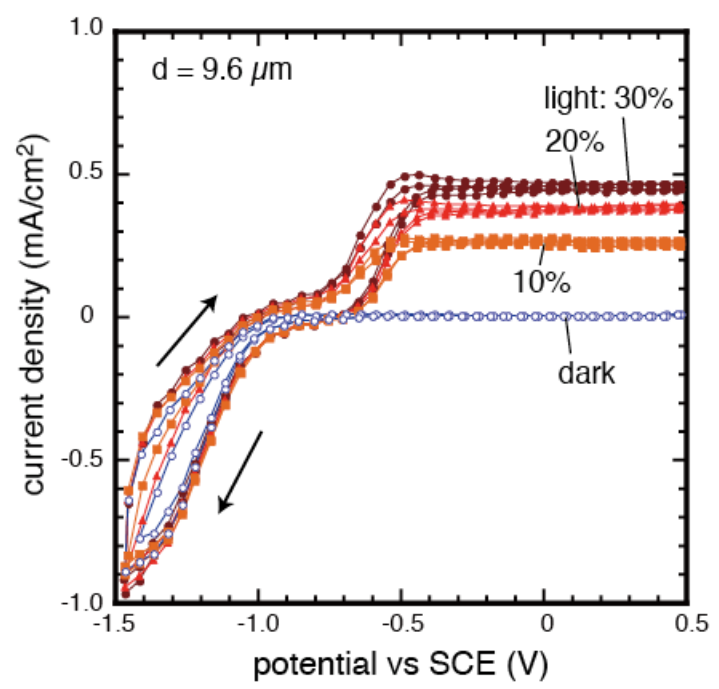

Figure 3: Cyclic voltammograms measured on InP porous electrode with pore depth, $d$, of $9.6 \mu \mathrm{m}$ in $\mathrm{K}_{3} \mathrm{Fe}(\mathrm{CN})_{6}$ electrolyte under various light conditions.
The anodic photocurrents of the various porous electrodes with different $d$ 's of 3.2, 4.8, 7.2, and 9.6 $\mu \mathrm{m}$ are compared in Fig. 4(a). In comparison, the maximum photocurrent observed for the planar electrode $(d=0 \mu \mathrm{m})$ was $0.003 \mathrm{~mA} / \mathrm{cm}^{2}$, which was very small and negligible in a range of $0.1 \mathrm{~mA} / \mathrm{cm}^{2}$. Such extremely small currents are probably because the measurements were conducted in a low-concentration electrolyte under a low-intensity light. On the other hand, the photocurrents of the InP porous electrodes were observed in this current range for all samples, as shown in Fig. 4(a). Furthermore, the anodic currents were found to increase with $d$ whereas the cathodic currents did not change very much.

Figure 4(b) plots the anodic photocurrents measured at the applied potential of $0 \mathrm{~V}$ as a function of the pore depth, $d$. As expected, the anodic photocurrents showed approximately linear relationships with $d$ because the anode efficiency proportionally increased as the surface area of the inside pore was enlarged as a linear function of $d$. Similar effects of the enlarged surface area have
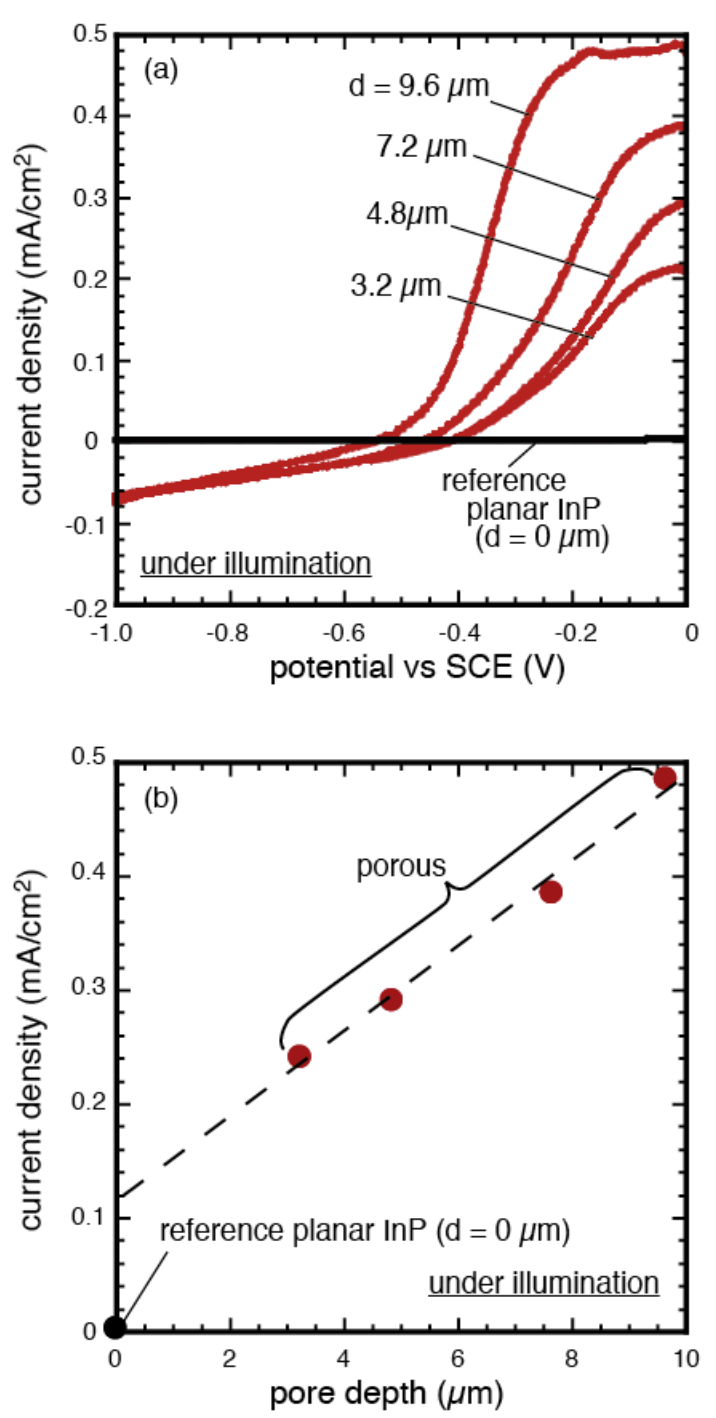

Figure 4: (a) Current-voltage $(I-V)$ characteristics of InP porous structures that have different pore depth, $d$, of 3.2 , 4.8, $7.29 .6 \mu \mathrm{m}$. (b) Correlation between anodic photocurrents measured at applied potential of $0 \mathrm{~V}$ and $d$. 
T. Sato et al., Thin Solid Films, 518(15), 2010, pp. 4399-4402.

been reported on the amperometric chemical sensor based on the InP porous structures [17]. However, the difference in the photocurrents between the porous electrodes and the reference planar electrode cannot be explained only by the effects of the enlarged surface area. As shown in Fig. 4(b), the extrapolation value given by the data of the porous electrodes was larger than the experimentally obtained value plotted at $d=0 \mu \mathrm{m}$ for the reference planar electrode.

These results suggest that the porous structures have a larger incident light absorption property than bulk InP. As shown in Figs. 2(a) and (b), the extremely low reflectance surfaces were obtained on the porous structures after the PEC etching, where the pores with the enlarged openings were vertically aligned. In such a case, the incident light goes through the porous layer by repeating the absorption on the pore walls due to an effective optical path longer than that of bulk InP. Another possible reason for the absorption enhancing is the optical transition involving both surface states [18] and quantum states [19] formed on the pore wall. These additional transitions give extra absorption in a wider energy range than the band gap of bulk InP. Thus, it can be concluded that the efficiency of a photon energy conversion is enhanced in the porous layer, resulting in the large anodic photocurrents increasing as the number of positive holes increases.

\section{Conclusion}

In conclusion, we have demonstrated that the surface reflectance of InP was significantly reduced by the formation of the porous structures. PEC etching after the pore formation very effectively reduced the reflectance to less than $0.1 \%$ in the visible light range and less than $0.4 \%$ in the UV and near-infrared ranges. Photoelectrochemical measurements on the porous structures revealed that the large anodic photocurrents are obtained on the samples that have low reflectance surfaces with deeper pores. These results can be explained by the two unique features of the InP porous structures: the large surface area inside pores, and the large photon absorption in the porous layer.

\section{Acknowledgments}

The work reported here was supported in part by a Grant-in-Aid for young scientists (A) - 21686028 from the Japanese Ministry of Education, Culture, Sports, Science, and Technology (MEXT) and by a Grant-in-Aid for Challenging Exploratory Research - 21656078, from the Japan Society for the Promotion of Science (JSPS).

\section{References}

[1] C. X. Shi, D. Grutzmacher, M. Stollenwerk, Q. K. Wang, K. Heime, IEEE Trans. Electron Devices 39 (1992) 1028.

[2] F. Zappa, A. Lacaita, S. Cova, P. Webb, Opt. Lett. 19 (1996) 846.

[3] A. Khan, A. Freundlich, J. Gou, A. Gapud, M. Imazumi, M. Yamaguchi, Appl. Phys. Lett. 90 (2007) 233111.

[4] M. F. Schubert, F. W. Mont, S. Chhajed, D. J. Poxson, J. K. Kim, E. F. Schubert, Opt. Exp. 16 (2008) 5290.

[5] P. Jenkins, G. A. Landis, N. S. Fatemi, D. Scheiman, X. Li, S. G. Bailey, Sol. Energy Mater. Sol. Cells 33 (1994) 125.

[6] N. G. Ferreira, D. Soltz, F. Decker, L. Cescato, J. Electrochem. Soc. 142 (1995) 1348.

[7] N. L. Dmitruk, O. Y. Borkovskaya, I. B. Mamontova, O. I. Mayeva, O. B. Yastrubchak, Thin Solid Films 364 (2000) 280.

[8] M. G. Boudreau, S. G. Wallace, G. Balcaitis, S. Murugkar, H. K. Haugen, P. Mascher, Appl. Optics 39 (2000) 1053.

[9] O. Maksimova, M. Munoz, N. Samarth, M.C. Tamargo, Thin Solid Films 467 (2004) 88.

[10] P. Thilakan, J. Kumar, This Solid Films 292 (1997) 50.

[11] T. Sato, T. Fujino, H. Hasegawa, Appl. Surf. Sci. 252 (2006) 5457.

[12] T. Sato, A. Mizohata, Electrochem. Solid State Lett. 11 (2008) H111.

[13] A. Theuwis, I. E. Vermeir, J. Electrochem. Soc. 146 (1999) 1172.

[14] P. Lautenschlager, M. Garriga, M. Cardona, Phys. Rev. B 36 (1987) 4813.

[15] L. L. Ma, Y. C. Zhou, N. Jiang, X. Lu, J. Shao, W. Lu, J. Ge, X. M. Ding, X. Y. Hou, Appl. Phys. Lett. 88 (2006) 171907.

[16] Z.-P. Yang, L. Ci, J. A. Bur, S.-Y. Lin, P. M. Ajayan, Nano Lett. 8 (2008) 446.

[17] T. Sato, A. Mizohata, N. Yoshizawa, T. Hashizume, Appl. Phys. Exp. 1 (2008) 051202.

[18] H. Fujikura, A. Liu, A. Hamamatsu, T. Sato, H. Hasegawa, Jpn. J. Appl. Phys. 39 (2000) 4616.

[19] T. Sato, T. Fujino, T. Hashizume, Electrochem. Solid State Lett. 10 (2007) H153. 